\title{
Structural partitioning of complex structures in the medium-frequency range. An application to an automotive vehicle
}

\author{
M. Kassem ${ }^{\text {a,b }}$, C. Soize ${ }^{a, *}$, L. Gagliardini ${ }^{b}$ \\ ${ }^{a}$ Université Paris-Est, Laboratoire Modélisation et Simulation Multi-Echelle, \\ MSME UMR 8208 CNRS, 5 Bd Descartes,77454 Marne-la-Vallee, France \\ ${ }^{\mathrm{b}}$ PSA Peugeot Citroen Route de Gisy, 78943 Velizy-Villacoublay, France
}

\begin{abstract}
In a recent work [Journal of Sound and Vibration 323 (2009) 849-863] the authors presented an energy-density field approach for the vibroacoustic analysis of complex structures in the low and medium frequency ranges. In this approach, a local vibroacoustic energy model as well as a simplification of this model were constructed. In this paper, firstly an extension of the previous theory is performed in order to include the case of general input forces and secondly, a structural partitioning methodology is presented along with a set of tools used for the construction of a partitioning. Finally, an application is presented for an automotive vehicle.
\end{abstract}

Key words: Vibroacoustic, Energy Analysis, stochastic computational Model, uncertainties, zone partitioning.

\section{Introduction}

The design process of automotive vehicles is increasingly concerned by the vibroacoustic performance which becomes an important criterion. Other design criteria such as the mass reduction and the cost reduction are additional constraints imposed to the vibroacoustic conception process. Presently, the vibroacoustic conception process, as for other domains, are more and more based on the use of numerical simulation with computational models. Today, the vibroacoustic computational models which are used are becoming very

\footnotetext{
* Corresponding author

Email address: christian.soize@univ-paris-est.fr (C. Soize).
} 
complex in order to represent the real systems. The objectif of the present work is to simplify the vibroacoustic analysis of complex structures such as an automotive vehicle.

The vibroacoustic analysis of an automotive vehicle is a very complicated task not only because of the complexity of the computational model itself but also because of the existence of a large number of noise sources and of observation positions. There can be more than 100 forces applied to the car body and up to 50 observation positions when considering noise and vibration. The analysis of such systems then requires to study a large number of Frequency Response Functions (FRF). Generally, such a task is very difficult. In addition to this difficulty, the presence of uncertainties in the computational models creates important differences between the numerical and the experimental results which increases the complexity of the analysis. These uncertainties should be taken into account in order to improve the quality of the computational models.

For the low and medium frequency vibroacoustic analysis of automotive vehicles, the most efficient numerical methods used nowadays are those consisting in solving the boundary value problem using the finite element method and the modal reduction methods (see for instance [1] for the computational models in the low and medium frequency ranges).

The objective of this introduction is not to give a state-of-the art of all the methods devoted to the vibroacoustic analyses of simple and complex vibroacoustic systems for the low, the medium and the high frequency ranges. We are only interested in methods based on a statistical energy formulation which have the capability to perform a low and medium frequency vibroacoustic analysis for real complex systems such as an automotive vehicle. In this context, this paper is not devoted to the classical deterministic time-harmonic vibroacoustic simulation methods which are mainly devoted to the low frequency range and which can be extended to the vibroacoustic analysis of automotive vehicles in the medium frequency-range as soon as uncertainties are taken into account in the computational vibroacoustic model ([2] [3] [4]).

It should be noted that the vibroacoustic analyses of automotive vehicles in the high-frequency band are of a great importance for automotive engineering and can generally be treated by using statistical energy analysis (SEA) [5] and [6], its extension and the diffuse field methods for which numerous papers have been published in the last decade (see for instance [7] for uncertainty modelling in SEA; [8], [9] and [10] for the extension of SEA model to subsystems with non-uniform modal energy distribution, for SEA substructuring using cluster analysis and for SEA subcomponent modelling of input parameters; [11], [12] and [13] for hybrid methods for the vibration analysis of complex vibroacoustic systems; [14], [15]) and [16] for diffuse field methods, energy transfer in builtup structures, vibrational energy variance in a random subsystem and hybrid 
local energy formulation; [17] for the ensemble statistics of the band-avaraged energy of random system; [18] and [19] for the response variance prediction in SEA of built up systems; [20] for the introduction of the energy mobility). These methods generally use a frequency or a space averaging and are efficient only in the high frequency range for which the modal density is high enough. In general, these methods cannot directly be used to perform the low and medium frequency vibroacoustic analysis of automotive vehicles. It should be noted that the Virtual SEA- FEA-based modeling of mid-frequency structureborne noise introduced in [21])is a statistical energy method which allows the mid-frequency band to be analyzed for complex system.

This paper is devoted to a statistical energy method allowing the medium frequency vibroacoustic analysis to be performed for automotive vehicles.

In a previous work [4], the authors have proposed a simplified calculation of the low and medium frequency responses of the vibroacoustic systems by using an energy-density field approach. A statistical computational model is used to construct a local vibroacoustic energy model deduced from a statistically averaging and not deduced from a frequency or a space averaging. The statistical computational model is constructed using the non parametric probabilistic approach introduced in [22] [23] in order to take into account both the system parameters uncertainties and the modelling errors which induce the model uncertainties. Such a non parametric probabilistic approach of uncertainties has been experimentally validated (see for instance [24] [25] [2] [3]). It has been shown that the vibroacoustic system is represented by "dimensionless power frequency response functions" which are slightly dependent of the frequency and of the direction of the excitation. A simplified local vibroacoustic energy model has also been proposed to represent the vibroacoustic system for a given set of excitations and for a given set of observed degrees of freedom (dofs), these two sets being sufficiently distant from each others. An appropriate error function has been introduced and the error induced by the introduction of such a simplified model has been evaluated.

It should be noted that the terminology "local" introduced above in the vibroacoustic energy models (local vibroacoustic energy model and simplified local vibroacoustic energy model) was not used in [4]. However, the authors think that it is important to clarify this point in order to emphasize the fact that the developed energy models give either a point-by-point description of the vibroacoustic system (local vibroacoustic energy model) or a local zone by local zone description of the vibroacoustic system (simplified local vibroacoustic energy model).

In the present paper, the authors present three new results. The first one is an extension of the energy formulation presented in [4] to the case of general input forces. The second one is the development of a structural partitioning 
methodology mainly devoted to the medium frequency range. The last one is an application to a complex automotive vehicle. It should be noted that the terminology "partitioning" is used instead of "substructuring".

In order to facilitate the reading of this paper, Section 2 is devoted to a brief summary of the construction introduced in [4] concerning the local vibroacoustic energy model and the simplified local vibroacoustic energy model. In Section 3, the extension of the local vibroacoustic energy formulation to the case of general input forces is presented. Section 4 deals with the structural partitioning methodology and its criteria. Finally, Section 5 is devoted to the application of the structural partitioning methodology to an automotive vehicle.

\section{Brief summary of the energy formulation}

In this section, a brief summary of the theory presented in [4] is given. This theory concerns the local vibroacoustic energy model and the simplified local vibroacoustic energy model.

\subsection{Construction of the local vibroacoustic energy model}

The details of the energy-density field approach are given in [4]. The main idea of this approach consists in replacing the usual set of the random frequency response functions relating the input forces to the random output velocities of the uncertain vibroacoustic system, by a new representation based on local energy quantities.

For a given set $\left\{j_{1}, \ldots, j_{\alpha}, \ldots, j_{r}\right\}$ of the $r$ dofs corresponding to the excited and observed dofs, the uncertain vibroacoustic system is represented by the following linear equation

$$
\mathbf{V}^{\alpha}(\omega)=[\mathbf{T}(\omega)] \mathbf{f}^{\alpha}(\omega)
$$

in which $[\mathbf{T}(\omega)]$ is the random complex square matrix valued Frequency Response Function (FRF) and where $\mathbf{V}^{\alpha}(\omega)$ is the complex random vector of the velocity responses. For $\alpha$ fixed in $\{1, \ldots, r\}$, let $t \mapsto \mathbf{f}^{\alpha}(t)$ be the function from $\mathbb{R}$ into $\mathbb{R}^{r}$ representing the excitation vector relative to the dof $j_{\alpha}$ and which is written as $\mathbf{f}^{\alpha}(t)=\left\{0, \ldots, f_{\alpha}^{\alpha}(t), \ldots, 0\right\}$. It is assumed that $\mathbf{f}^{\alpha}(-t)=\mathbf{f}^{\alpha}(t)$ and that $\mathbf{f}^{\alpha}$ is square integrable on $\mathbb{R}$. In Eq. (1), $\mathbf{f}^{\alpha}(\omega)=\int_{-\infty}^{+\infty} e^{-i \omega t} \mathbf{f}^{\alpha}(t) d t$ is the Fourier transform of $\mathbf{f}^{\alpha}(t)$ which is a real function such that $\mathbf{f}^{\alpha}(-\omega)=\mathbf{f}^{\alpha}(\omega)$ and which is written as $\mathbf{f}^{\alpha}(\omega)=\left\{0, \ldots, f_{\alpha}^{\alpha}(\omega), \ldots, 0\right\}$. 
On the other hand, the energy-density field approach represents the vibroacoustic system by the means of a random real matrix valued Power Frequency Response Function $(\mathrm{PFRF})[\mathcal{E}(\omega)]$ relating a random vector valued input power density function $\boldsymbol{\pi}_{i n}(\omega)$ to what is called the local response vector valued random power density function $\boldsymbol{\pi}^{R}(\omega)$ such that

$$
\boldsymbol{\pi}^{R}(\omega)=[\mathcal{E}(\omega)] \boldsymbol{\pi}_{i n}(\omega)
$$

In Eq. (2), the PFRF $[\mathcal{E}(\omega)]$ is a dimensionless quantity which is defined by

$$
[\mathcal{E}(\omega)]=[\boldsymbol{Y}(\omega)]^{-1}[\boldsymbol{H}(\omega)][\boldsymbol{Y}(\omega)]^{-1}
$$

where $[\boldsymbol{Y}(\omega)]$ is the random diagonal matrix of the driving point mobilities which is written as,

$$
[\boldsymbol{Y}(\omega)]_{\alpha \beta}=\left\{\begin{array}{cc}
\operatorname{Re}\left\{[\boldsymbol{T}(\omega)]_{\alpha \alpha}\right\} & \text { si } \alpha=\beta \\
0 & \text { si } \alpha \neq \beta
\end{array} .\right.
$$

In Eq. (4), the random matrix $[\boldsymbol{H}(\omega)]$ is defined by $[\boldsymbol{H}(\omega)]_{\beta \alpha}=\left|[\boldsymbol{T}(\omega)]_{\beta \alpha}\right|^{2}$. In the energy approach, two other important quantities representing the excitation and the response of the energy model are introduced. The first quantity is the vector-valued spectral density function $\boldsymbol{s}^{f}(\omega)=\left(s_{1}^{f}(\omega), \ldots, s_{r}^{f}(\omega)\right)$ of the input forces in which $s_{\alpha}^{f}(\omega)=(1 / 2 \pi) f_{\alpha}^{\alpha}(\omega)^{2}$. As explained above, $f_{\alpha}^{\alpha}(\omega)$ is the component $\alpha$ of the force vector $\mathbf{f}^{\alpha}(\omega)$. The second quantity is the random vector-valued spectral density function $\boldsymbol{S}^{v}(\omega)$ of the output response such that $\boldsymbol{S}^{v}(\omega)=2[\boldsymbol{H}(\omega)] \boldsymbol{s}^{f}(\omega)$. The random input power is calculated by

$$
\boldsymbol{\pi}_{i n}(\omega)=2[\boldsymbol{Y}(\omega)] \boldsymbol{s}^{f}(\omega)
$$

The vibroacoustic energy model is obtained in taking the mathematical expectation of the stochastic energy formulation. Let $E$ be the mathematical expectation. One can thus define the statistically averaged quantities $[\underline{T}(\omega)]=$ $E\{[\boldsymbol{T}(\omega)]\},[\underline{Y}(\omega)]=E\{[\underline{\boldsymbol{Y}}(\omega)]\}$, the mean value $[\underline{\mathcal{E}}(\omega)]$ of the random PFRF which is such that

$$
[\underline{\mathcal{E}}(\omega)]=E\left\{[\boldsymbol{Y}(\omega)]^{-1}[\boldsymbol{H}(\omega)][\boldsymbol{Y}(\omega)]^{-1}\right\} \quad,
$$

and the mean value $\underline{\boldsymbol{s}}^{v}(\omega)^{r e f}=E\left\{\boldsymbol{S}^{v}(\omega)\right\}$ of the vector-valued spectral density function of the random output response $\boldsymbol{S}^{v}(\omega)$ such that

$$
\underline{\boldsymbol{s}}^{v}(\omega)^{r e f}=2 E\left\{[\boldsymbol{Y}(\omega)][\mathcal{E}(\omega)][\boldsymbol{Y}(\omega)] \boldsymbol{s}^{f}(\omega)\right\} .
$$

It is important to mention that all the equations of the vibroacoustic energy model can be rewritten in the local coordinates defined by the principal directions of the matrix defined as the mean values of the random local mobilities. In this case, the new equations are called the equations of the local vibroacoustic energy model. For more details regarding these local coordinates, the 
reader is referred to [4]. Below it is assumed that all the equations are written in these local coordinates and one will then consider the local vibroacoustic energy model.

\subsection{Simplified local vibroacoustic energy model}

The simplified local vibroacoustic energy model is then constructed using the following assumption. Let $J=\left\{k_{q}, q=1, \ldots, \mu\right\}$ be the set of the $\mu$ given excited dofs and let $O=\left\{j_{p}, p=1, \ldots, \nu\right\}$ be the set of the $\nu$ given observed dofs. It is assumed that these excited dofs and these observed dofs are geometrically distant. The mean value of the random matrix valued PFRF can be approximated by a positive valued deterministic scalar quantity $\underline{e}_{O J}$ which is such that, for each $\omega$ in the frequency band of analysis $\mathbb{B}$, for each $p=1, \ldots, \nu$ and for each $q=1, \ldots, \mu$, one can write

$$
[\underline{\mathcal{E}}(\omega)]_{j_{p} k_{q}} \simeq \underline{e}_{O J}(\omega)
$$

Using Eq. (8) and from Eq. (7), the following approximation can be deduced,

$$
\underline{\mathbf{s}}^{v}(\omega)_{j_{p}}^{a p p}=\underline{e}_{O J}(\omega)[\underline{Y}(\omega)]_{j_{p} j_{p}} \underline{\pi}_{i n, J},
$$

where $\underline{\pi}_{i n, J}=\sum_{q=1}^{\mu} \underline{\pi}_{i n}^{q}$ is the mean value of the random total input power relative to set $J$. It has been proven in [4] that such an approximation can be used for complex vibroacoustic systems. It is however important to note that Eq. (9) is not used to calculate $\underline{e}_{O J}(\omega)$. In fact, $\underline{e}_{O J}(\omega)$ is calculated using the reference value $\underline{\mathbf{s}}^{v}(\omega)^{\text {ref }}$ given by Eq. (7) (without any approximation) as

$$
\underline{e}_{O J}(\omega)=\frac{\sum_{p=1}^{\nu} \underline{\mathbf{s}}^{v}(\omega)_{j_{p}}^{r e f}}{\underline{\pi}_{i n, J} \sum_{p=1}^{\nu}[\underline{Y}(\omega)]_{j_{p} j_{p}}} .
$$

\section{Random input power calculation for general input forces}

In Section 2, the theory has been developed for particular input forces $\mathbf{f}^{\alpha}(\omega)$ where $\mathbf{f}^{\alpha}(\omega)=\left\{0, \ldots, f_{\alpha}^{\alpha}(\omega), \ldots, 0\right\}$ is a real vector with only one non zero component. Below, we propose an extension of the theory for which the input power is calculated for general input forces written as $\mathbf{f}^{\alpha}(\omega)=\left\{f_{1}^{\alpha}(\omega), \ldots\right.$, $\left.f_{\alpha}^{\alpha}(\omega), \ldots, f_{r}^{\alpha}(\omega)\right\}$ where $\mathbf{f}^{\alpha}(\omega)$ is a complex vector with at least two non zero components. The results presented in this section allow the extension of the application range of the energy-density field approach. 
In reference [4], the random input power density is given by

$$
\pi_{i n}^{\alpha}(\omega)=\frac{1}{\pi} \operatorname{Re}\left\{[\boldsymbol{T}(\omega)]_{\alpha \alpha}\right\} f_{\alpha}^{\alpha}(\omega)^{2}
$$

Equation (11) were derived assuming that the real input forces were written as $\mathbf{f}^{\alpha}(\omega)=\left\{0, \ldots, f_{\alpha}^{\alpha}(\omega), \ldots, 0\right\}$. Thus, in order to exactly calculate the random input power for general input forces $\mathbf{f}^{\alpha}(\omega)=\left\{f_{1}^{\alpha}(\omega), \ldots, f_{\alpha}^{\alpha}(\omega), \ldots, f_{r}^{\alpha}(\omega)\right\}$, a base change into a new basis is introduced. This basis is constructed using the eigenvectors of the mean mobility matrix associated with all the excited dofs.

Let $\left[T^{i n}(\omega)\right]$ be the square sub-matrix of $E\{[\boldsymbol{T}(\omega)]\}$ corresponding to the $r$ excited dofs. The real symmetric matrix $\left[T_{R}^{i n}(\omega)\right]$ corresponding to the real part of $\left[T^{i n}(\omega)\right]$ can be written as

$$
\left[T_{R}^{i n}(\omega)\right]=\left[\phi^{i n}(\omega)\right]\left[\mathcal{T}_{R}^{i n}(\omega)\right]\left[\phi^{i n}(\omega)\right]^{T},
$$

where $\left[\mathcal{T}_{R}^{i n}(\omega)\right]$ is the real diagonal matrix containing the eigenvalues of $\left[T_{R}^{i n}(\omega)\right]$ and where $\left[\phi^{i n}(\omega)\right]$ is the matrix made up of the corresponding eigenvectors. The family of these eigenvectors constitutes the basis which diagonalizes $\left[T_{R}^{i n}(\omega)\right]$. Introducing hermitian scalar product such as $\ll x, y \gg=\sum_{j} x_{j} \overline{y_{j}}$, the mean input power density is written as

$$
\begin{aligned}
\underline{\pi}_{i n}^{\alpha}(\omega)=\frac{1}{\pi} \operatorname{Re}\left\{\ll \boldsymbol{f}^{\alpha}(\omega),\left[\phi^{i n}(\omega)\right][\right. & \left.\mathcal{T}_{R}^{i n}(\omega)\right]\left[\phi^{i n}(\omega)\right]^{T} \boldsymbol{f}^{\alpha}(\omega) \gg \\
& \left.-i \ll \boldsymbol{f}^{\alpha}(\omega),\left[T_{I}^{i n}(\omega)\right] \boldsymbol{f}^{\alpha}(\omega) \gg\right\},
\end{aligned}
$$

where $\left[T_{I}^{i n}(\omega)\right]$ is the imaginary part of $\left[T^{i n}(\omega)\right]$. It can easily be proven that Eq. (13) can be written as

$\underline{\pi}_{i n}^{\alpha}(\omega)=\frac{1}{\pi} \operatorname{Re}\left\{\sum_{\beta}\left[\mathcal{T}_{R}^{i n}(\omega)\right]_{\beta \beta}\left|\mathcal{F}_{\beta}^{\alpha}(\omega)\right|^{2}\right\}+\frac{1}{\pi} \operatorname{Im}\left\{\ll\left[T_{I}^{i n}(\omega)\right] \boldsymbol{f}^{\alpha}(\omega), \boldsymbol{f}^{\alpha}(\omega) \gg\right\}$,

in which $\mathcal{F}^{\alpha}(\omega)=\left[\phi^{i n}(\omega)\right]^{T} \boldsymbol{f}^{\alpha}(\omega)$. Since $\ll\left[\mathcal{T}_{I}^{i n}(\omega)\right] \boldsymbol{f}^{\alpha}(\omega), \boldsymbol{f}^{\alpha}(\omega) \gg$ is an hermitian form, then $\ll\left[\mathcal{T}_{I}^{i n}(\omega)\right] \boldsymbol{f}^{\alpha}(\omega), \boldsymbol{f}^{\alpha}(\omega) \gg$ is a real number and thus, its imaginary part is equal to zero. The mean input power density can thus be written as

$$
\underline{\pi}_{i n}^{\alpha}(\omega)=\frac{1}{\pi} \sum_{\beta=1}^{r}\left[\mathcal{T}_{R}^{i n}(\omega)\right]_{\beta \beta}\left|\mathcal{F}_{\beta}^{\alpha}(\omega)\right|^{2}
$$

One can notice that Eq. (15) is the same as the mean value of Eq. (11) expressed for the $r$ excited dofs. Thus, in the new introduced basis which diagonalizes the matrix $\left[T_{R}^{i n}(\omega)\right]$, one can calculate $\underline{\pi}_{i n}^{\alpha}(\omega)$ using Eq. (15) for general input forces. 


\section{Structural partitioning methodology and criterion}

The construction of the simplified local vibroacoustic energy model introduced in Section 2.2 is based on the observation of the homogenous vibroacoustic behaviour of the vibroacoustic energy model throughout a given zone. The objective of this section is to introduce (1) a structural partitioning methodology and (2) the partitioning tools to construct a structural partitioning.

The main objective of the structural partitioning methodology is to define a structural partitioning in terms of zones called structural zones for which one can use the approximation introduced by Eq. (8) between two structural zones. It should be noted that the acoustic cavity is considered as a structural zone for the structural partitioning methodology.

It should be noted that the strategy of dynamic substructuring consists in replacing the global model of the structure by an assemblage of substructures. Presently, the partitioning methodology is not a dynamic substructuring. The vibroacoustic analysis is always performed with the global vibroacoustic system. The post processing of the results obtained are then used to construct the structural partitioning.

It is necessary to introduce error functions which have the capability to estimate the error introduced by the use of this approximation. One thus introduces the two error functions $\varepsilon_{\mathcal{E}}(\omega)_{j_{p} k_{q}}$ and $\Delta(\omega)_{j_{p}, J}$. The first one is a matrix valued function which measures the distance between $[\underline{\mathcal{E}}(\omega)]_{j_{p} k_{q}}$ and $\underline{e}_{O J}(\omega)$ and which is such that, for all $j_{p}$ in $O, k_{q}$ in $J$ and $\omega$ in $\mathbb{B}$, one has

$$
\varepsilon_{\mathcal{E}}(\omega)_{j_{p} k_{q}}=\left|\left[\mathrm{dB}(\omega)^{r e f}\right]_{j_{p} k_{q}}-\left[\mathrm{dB}(\omega)^{p a r t}\right]_{j_{p} k_{q}}\right|
$$

where

$$
\begin{gathered}
{\left[\mathrm{dB}(\omega)^{r e f}\right]_{j_{p} k_{q}}=10 \log _{10}[\underline{\mathcal{E}}(\omega)]_{j_{p} k_{q}},} \\
{\left[\mathrm{~dB}(\omega)^{\text {part }}\right]_{j_{p} k_{q}}=10 \log _{10}\left[\mathbb{E}_{O J}(\omega)^{\text {part }}\right]_{j_{p} k_{q}},}
\end{gathered}
$$

with $\left[\mathbb{E}_{O J}(\omega)^{\text {part }}\right]_{j_{p} k_{q}}=\underline{e}_{O J}(\omega)$.

The second one measures the distance between $\underline{\boldsymbol{s}}^{v}(\omega)_{j_{p}}^{\text {part }}$ and $\underline{\boldsymbol{s}}^{v}(\omega)_{j_{p}}^{r e f}$, and is such that, for all $j_{p}$ in $O$ and for all $\omega$ in $\mathbb{B}$, one has

$$
\Delta(\omega)_{j_{p}, J}=\left|\underline{s}^{v}(\omega)_{j_{p}}^{r e f}-\underline{s}^{v}(\omega)_{j_{p}}^{p a r t}\right|
$$

The above error function can thus be used to estimate the relevance of a given structural partitioning. However, as it is easier to interpret the values of a scalar valued function than those of a vector valued function, one introduces the scalar valued centered quadratic mean criterion $C_{O J}(\omega)$ in $\log$ scale defined 
as the centered quadratic mean of the vector valued function $\Delta(\omega)_{j_{p}, J}$ such as

$$
C_{O J}(\omega)=10 \log _{10}\left(\frac{1}{\nu} \sum_{p=1}^{\nu}\left\{\Delta(\omega)_{j_{p}, J}\right\}^{2}-\left\{\frac{1}{\nu} \sum_{p=1}^{\nu} \Delta(\omega)_{j_{p}, J}\right\}^{2}\right)
$$

The function $\omega \mapsto C_{O J}(\omega)$ is thus used as a structural partitioning criterion depending on the frequency.

The construction of an effective structural partitioning of a given structure is done using these structural partitioning tools using the following methodology:

(1) An initial partitioning of the vibroacoustic system is chosen in terms of components of the vibroacoustic system (for an automotive vehicle, the components consists of the wind shield, the roof, the internal cavity, etc).

(2) The partitioning criterion $\omega \mapsto C_{O J}(\omega)$ is then plotted for a given excited structural zone $J$ and for a given observed structural zone $O$. The value of the partitioning criterion helps to find the value $\omega^{*}$ of the frequency such that for all $\omega \geq \omega^{*}$, the approximation introduced by the simplified local vibroacoustic energy model can be used with a good approximation. In the case for which no $\omega^{*}$ exists in the frequency band of analysis $\mathbb{B}$, it is necessary to redefine the components of the partitioning. This step is done for all possible combinations of excited zone and of observed zone chosen among the components.

(3) In order to redefine the partitioning, one can observe the color plot of the matrix valued error function $(p, q) \mapsto \varepsilon_{\mathcal{E}}(\omega)_{j_{p} k_{q}}$ for the zone of interest in order to determine the dof which have to be removed from the zone and introduced in another zone. These two steps can then be repeated in order to evaluate the new partitioning. It is important to note that the present step depends on a "visual" evaluation of the color plot and that it would be much preferable to use an automatic numerical algorithm. Such an algorithm has not been developed in this work because such a development is a straightforward engineering application.

\section{Application to an automotive vehicle}

In this section, the partitioning methodology presented in the previous section is applied to an automotive vehicle structure coupled with its internal acoustic cavity. The finite element model consists of 1042851 dofs for the structure and of 9157 dofs for the acoustic cavity. The two meshes are considered compatible on the vibroacoustic coupling interface. The analysis has been performed using the translational dofs of 28 structural nodes $(28 \times 3=84$ dofs $)$ and 12 acoustic nodes (12 dofs). One then has a total of 96 dofs. Figure 1 shows the finite 
element models of the structure and of the acoustic cavity with the positions of the selected excitation and observation nodes.
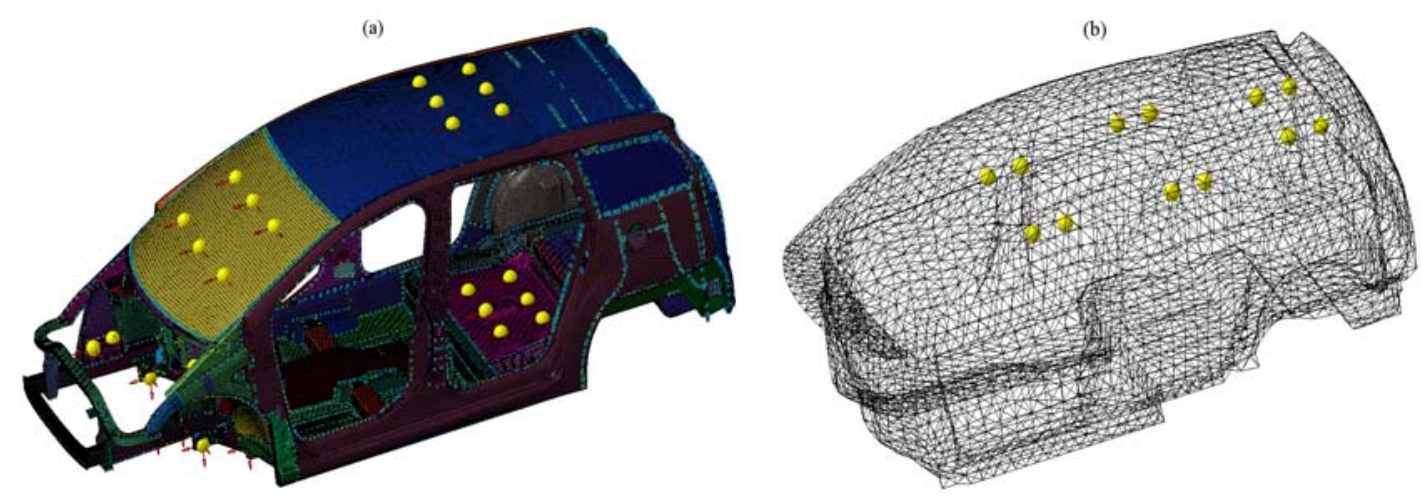

Figure 1. Finite element mesh of the structure consisting of 1042851 dofs with 28 excitation and observation nodes (a) and of the acoustic cavity consisting of 9157 dofs with 12 excitation and observation nodes (b).

The 28 structural nodes consist of, 6 nodes on the roof, 6 nodes on the wind shield, 6 nodes on the rear seat panel, 6 nodes on the front suspension attachement points and 4 nodes on the engine supports attachement points. Unit force excitations have been applied to all the structural dofs and unit acoustic sources have been placed at all the acoustic dofs. The observation dofs are the same as the excitation dofs.

\subsection{Calculation of the input power associated with a set of general input forces}

In this section, the input power associated with a set of general input forces applied to the engine support attachement points is calculated using section 3. The set of input forces has been measured by the car manufacturer on a test rig allowing real operating conditions. Figure 2 shows a comparison of the input power calculated with section 3 compared to the input power calculated using Eq. (5). This figure shows that the input power calculation cannot be simplified using Eq. (5) when the excitation forces correspond to general input forces. Above $110 \mathrm{~Hz}$, the difference between the input power calculated with section 3 and the input power calculated using Eq. (5) increases because, above this frequency, there are no dominant components in the vector valued force applied to the structure. 
(a)

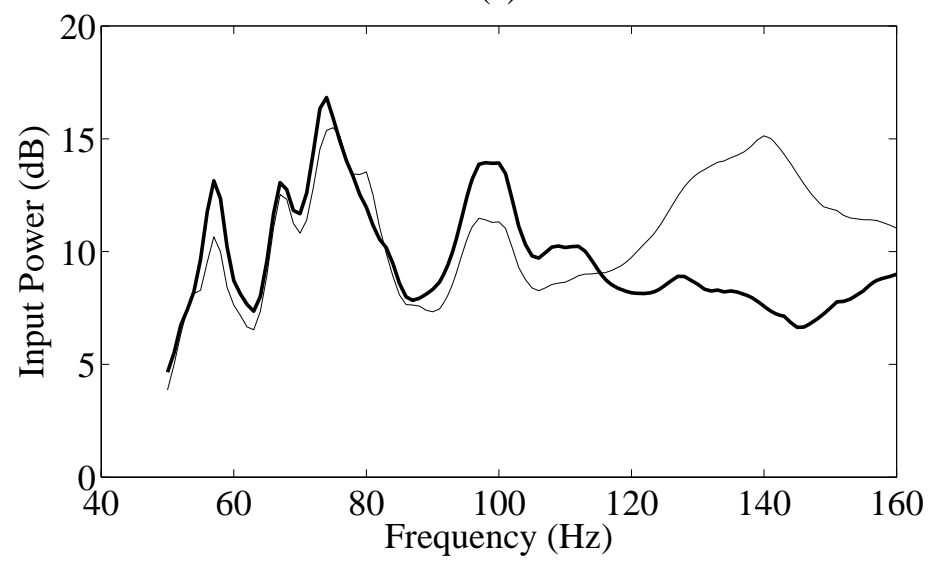

Figure 2. Graphs of the input power computed with section 3 (thick line) compared to the input power calculated using Eq. (5)(thin line)

\subsection{Construction of the partitioning of the automotive vehicle}

As mentioned above, for the application of the partitioning methodology, an initial partitioning is defined using the different structural components of the car body. First, the partitioning of the entire vibroacoustic system is studied. For this case, the excitation set $J$ contains all the 84 structural dofs $(\mu=84)$ and the set $O$ contains all the 12 acoustic dofs $(\nu=12)$. Figure 3 shows images of the error matrix $\varepsilon_{\mathcal{E}}(\omega)$ defined by Eq. (16), at the four frequencies $50 \mathrm{~Hz}$, $100 \mathrm{~Hz}, 150 \mathrm{~Hz}$ and $300 \mathrm{~Hz}$. The matrix $\varepsilon_{\mathcal{E}}(\omega)$ is a $(\mu+\nu \times \mu+\nu)$ matrix whose columns and rows related to the same set of dofs corresponding to the union of the dofs belonging to the sets $J$ and $O$. From figure 3, one can notice that the matrix $\varepsilon_{\mathcal{E}}(\omega)$ has a block diagonal structure. The error value is very high on the two blocks on the diagonal which represent the dofs of the structure (bigger block) and the dofs of the acoustic cavity (smaller block). For these two blocks, the excitation and the observation nodes are geometrically located in the same zone.

The error on the extra-diagonal blocks, corresponding to the $\mu$ excitation dofs and the $\nu$ observation dofs (or vice-versa by reciprocity), is relatively low starting at $50 \mathrm{~Hz}$. The detailed analysis performed with respect to the frequency sampling has shown that this low value is however not homogeneous throughout the extra-diagonal blocks until approximately $90 \mathrm{~Hz}$. This value of the frequency is coherent with figure 5 (see the explanation related to this figure).

One can then conclude that the dofs of the acoustic cavity are fully separated from those of the structure starting at low frequencies, whereas, they can be considered as a unique zone above $90 \mathrm{~Hz}$. 
(a)

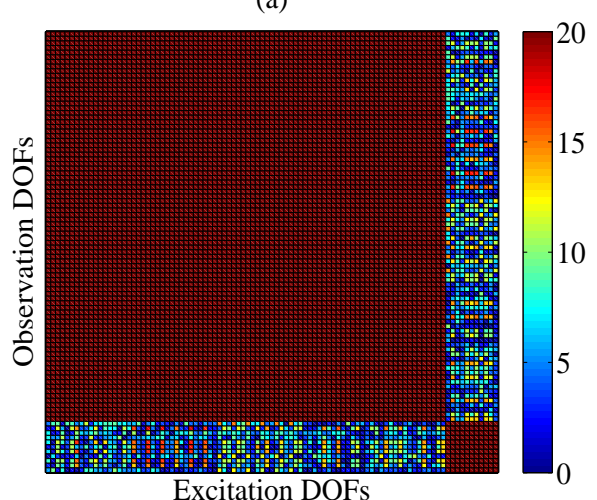

(c)

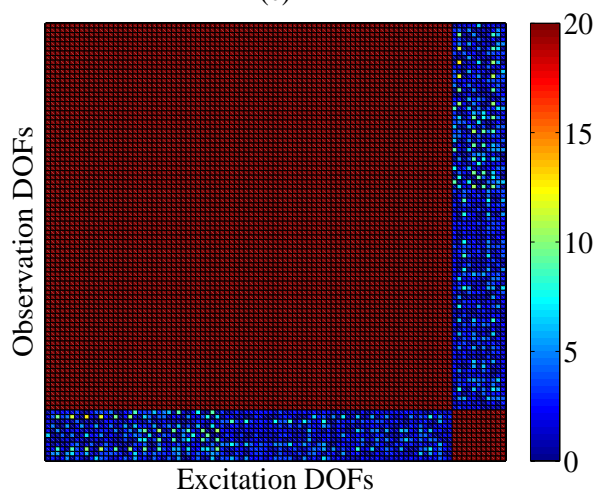

(b)

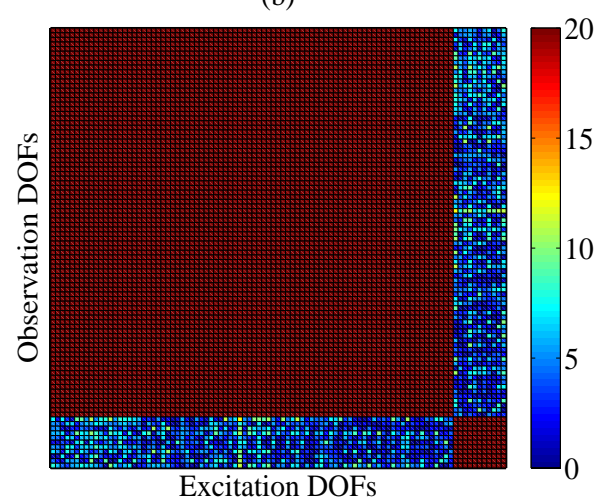

(d)

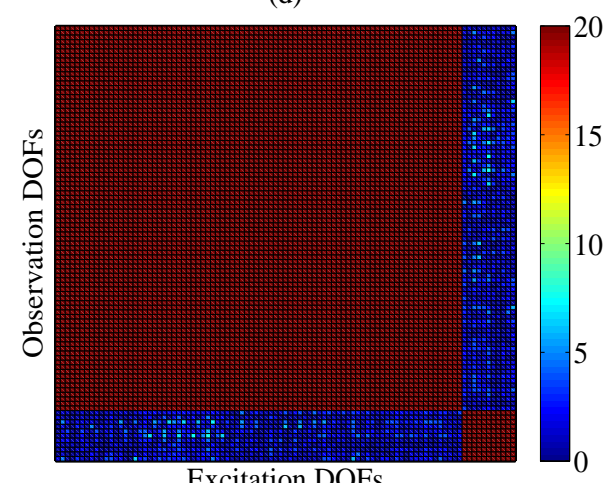

Excitation DOFs

Figure 3. Images of the error matrix $\varepsilon_{\mathcal{E}}(\omega)$. The excitations are applied to all the 84 dofs $(\mu=84)$ of the structure. The observations are all dofs of the acoustic cavity $(\nu=12)$. The frequencies are $50 \mathrm{~Hz}(\mathrm{a}), 100 \mathrm{~Hz}(\mathrm{~b}), 150 \mathrm{~Hz}$ (c) and $300 \mathrm{~Hz}$ (d).

One now considers the partitioning of the structure. Different excited structural zones and observed structural zones have been studied. For example, figure 4 shows images of the matrix $\varepsilon_{\mathcal{E}}(\omega)$. The excitation set $J$ contains the 30 dofs of the 10 nodes located in the front suspension and the engine supports attachement points $(\mu=30)$. The observation set $O$ contains the 6 dominant principal dofs of the 6 nodes located in the roof $(\nu=6)$. It is important to note that the dominant principal dofs are close to the normal dofs. For more details about the principal directions and about the dominant principal dofs of flexible structural elements (thin structural elements), the reader is referred to [4].

From figure 4, it can seen that the two blocks on the diagonal of the matrix show the highest error values. Moreover, it can also be seen that the two extra diagonal blocks, which are identical by reciprocity, have a low error value which becomes homogeneous for all the dofs inside these extra diagonal blocks starting at approximately $90 \mathrm{~Hz}$. It can then be concluded that the dofs that have been chosen on the roof are part of the same zone starting at this frequency. This zone (the roof) is separated from the front suspension 
zone and from the engine supports zones. In order to confirm this conclusion, the partitioning criterion presented in section 4 has been calculated for the configuration corresponding to figure 4 . Figure 5 displays the graph of this partitioning criterion as a function of the frequency.

(a)

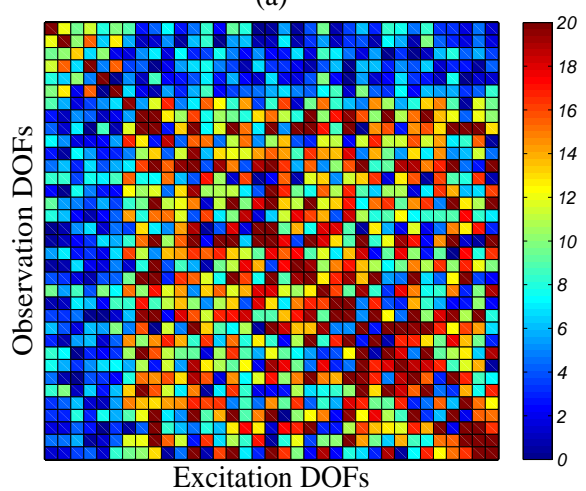

(c)

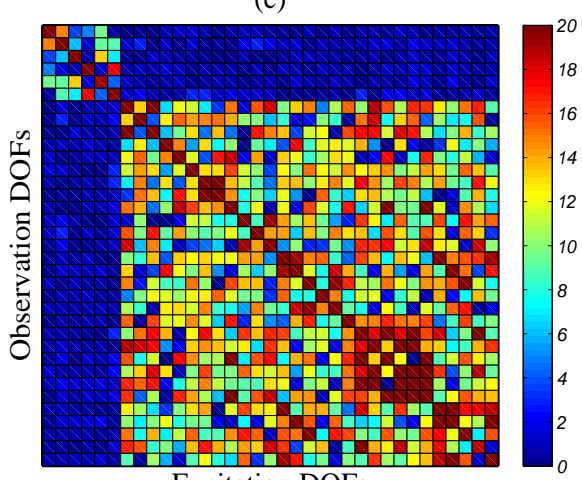

Excitation DOFs (b)

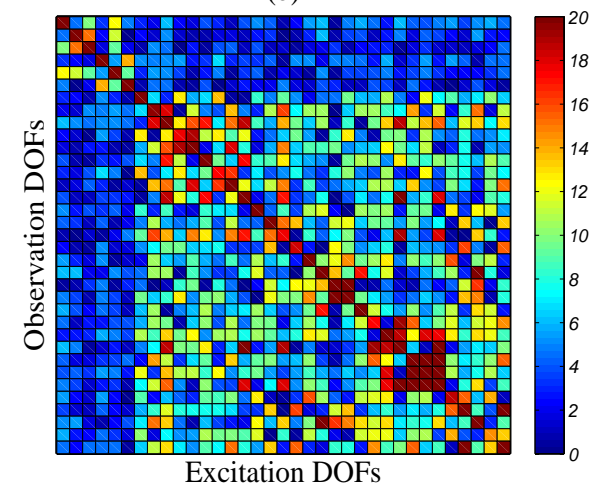

(d)

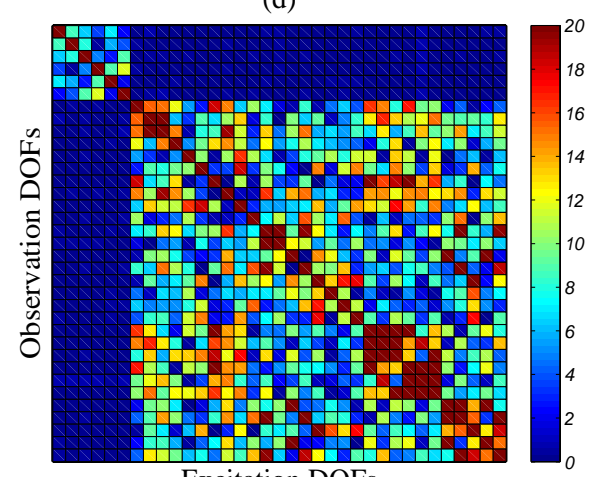

Excitation DOFs

Figure 4. images of the matrix $\varepsilon_{\mathcal{E}}(\omega)$. The excitation set $J$ contains the 30 dofs of the 10 nodes located in the front suspension and the engine supports attachement points $(\mu=30)$. The observation set $O$ contains the 6 dominant principal dofs of the 6 nodes located in the roof $(\nu=6)$. The frequencies are $50 \mathrm{~Hz}(\mathrm{a}), 100 \mathrm{~Hz}(\mathrm{~b})$, $150 \mathrm{~Hz}(\mathrm{c})$ and $300 \mathrm{~Hz}(\mathrm{~d})$.

From figure 5, it can be seen that the value of the partitioning criterion $C_{O J}$ is less than $2 \mathrm{~dB}$ above $90 \mathrm{~Hz}$. This indicates that, starting at this frequency, the simplified model can be used for the roof. This confirms the conclusion previously made based on the observation of the images of the error matrix $\varepsilon_{\mathcal{E}}(\omega)$

The sensitivity of the partitioning criterion to a given structural zones have also been tested. To do so, the observed structural zone corresponding to the configuration associated with figure 4 has been spatially extended to include the wind shield dofs. Figure 6 displays the images of $\varepsilon_{\mathcal{E}}(\omega)$ corresponding to this new configuration at $50 \mathrm{~Hz}, 100 \mathrm{~Hz}, 150 \mathrm{~Hz}$ and300 Hz. Figure 7 shows 
the partitioning criterion $C_{O J}$ as a function of the frequency corresponding to the same configuration.

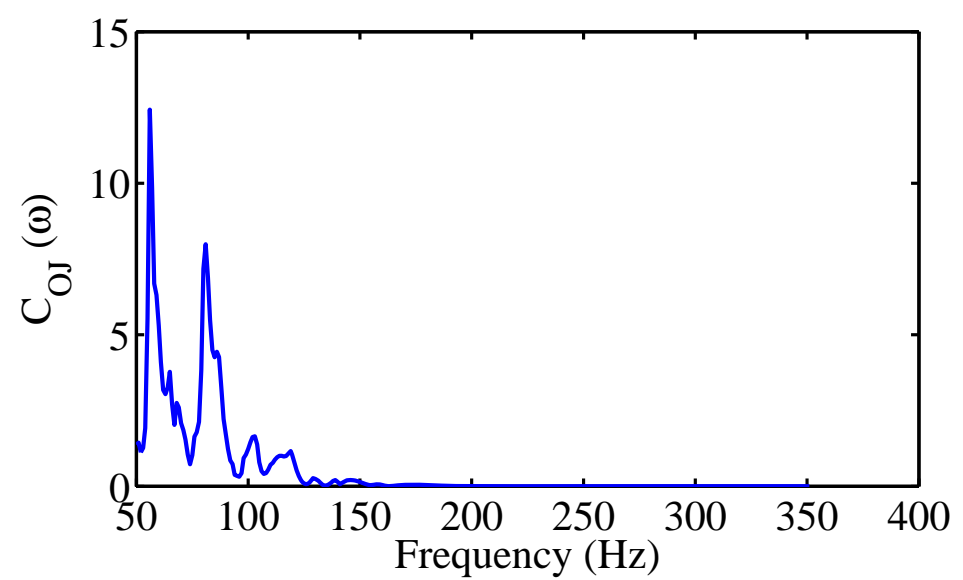

Figure 5. Graph of the partitioning criterion represented by the function $\omega \mapsto C_{O J}(\omega)$ corresponding to the configuration associated with figure 4

The value of the partitioning criterion shown in figure 7 is relatively high compared to that shown in figure 5. This is because the dofs of the wind shield are coupled with those of the front suspension and the engine supports attachement points. This can also be seen from the images of the error matrix $\varepsilon_{\mathcal{E}}(\omega)$ for which there is a coupling between the wind shield dofs (second diagonal block from the top) and those of the front suspension and the engine supports attachement points (last diagonal block from the top). This shows that the partitioning criterion is sensitive to the coupling between the excited zone and the observed zone.

In practice, one is interested in observing the images of the error matrix $\varepsilon_{\mathcal{E}}(\omega)$ as a function of the frequency for all the dofs chosen for the analysis. This observation helps to determine the value of the frequency at which each structural partition is separated from the entire structure as well as the frequency at which the structural partitioning becomes stable. Figure 8 displays the images of the assembled matrix $\varepsilon_{\mathcal{E}}(\omega)$ for all the chosen structural dofs at $50 \mathrm{~Hz}$, $100 \mathrm{~Hz}, 150 \mathrm{~Hz}$ and $300 \mathrm{~Hz}$.

One can observe 5 blocks on the diagonal of the assembled matrix. The blocks represent, from top to bottom, the roof, the wind shield, the rear seat panel, the left engine supports and the front suspension attachement dofs and, the right engine supports and the front suspension attachement dofs. Again, the error is relatively low for the extra diagonal blocks showing the relevance of the partition. The observation of this assembled matrix as a function of the frequency shows that the rear seat panel is separated from the other components of the structure starting at $50 \mathrm{~Hz}$ (the starting frequency of the band 
of analysis). The roof is then separated starting at $90 \mathrm{~Hz}$. The wind shield is separated starting at approximately $160 \mathrm{~Hz}$. Finally, the engine supports and the front suspension attachement dofs are separated into left and right dofs (with respect to the car structure) above $260 \mathrm{~Hz}$.

(a)

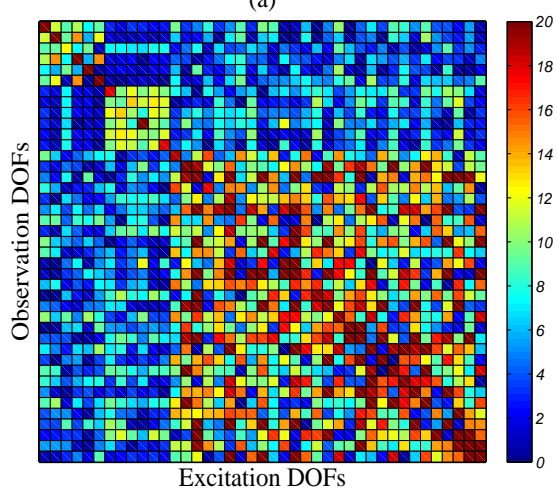

(c)

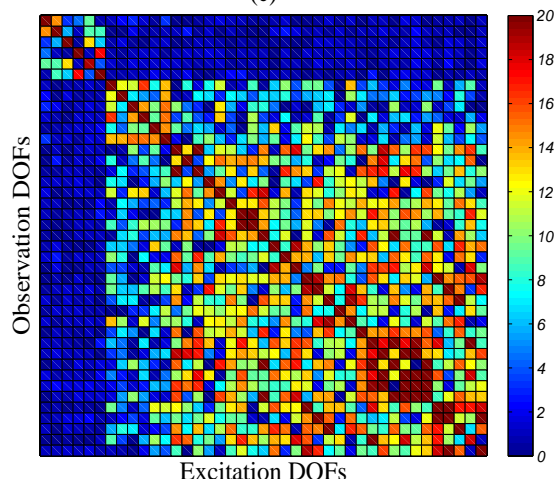

(b)

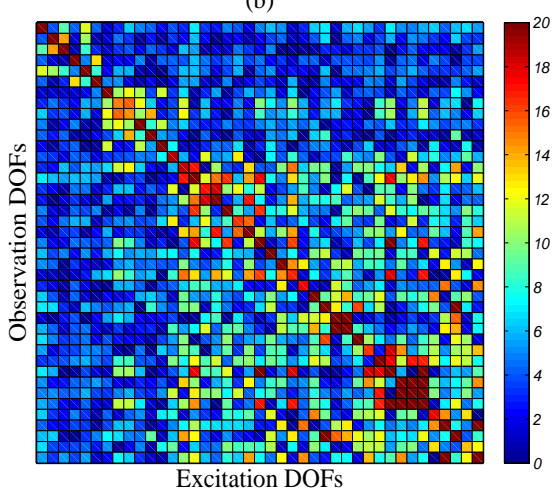

(d)

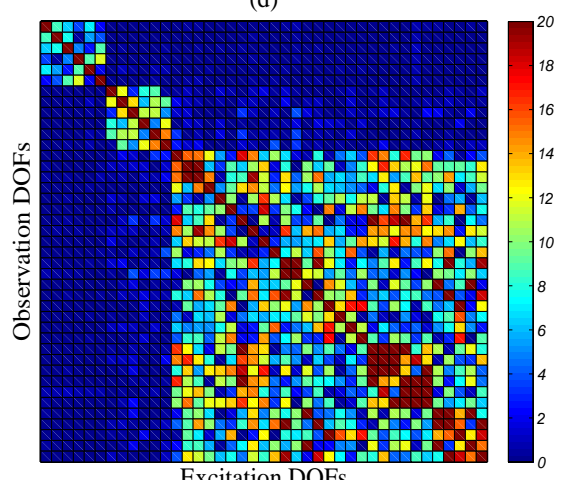

Figure 6. images of the matrix $\varepsilon_{\mathcal{E}}(\omega)$. The excitation set $J$ contains the 30 dofs of the 10 nodes located in the front suspension and the engine supports attachement points $(\mu=30)$. The observation set $O$ contains the 12 dominant principal dofs of the 12 nodes located in the roof and the wind shield $(\nu=12)$. The frequencies are $50 \mathrm{~Hz}$ (a), $100 \mathrm{~Hz}$ (b), $150 \mathrm{~Hz}$ (c) and $300 \mathrm{~Hz}$ (d). 


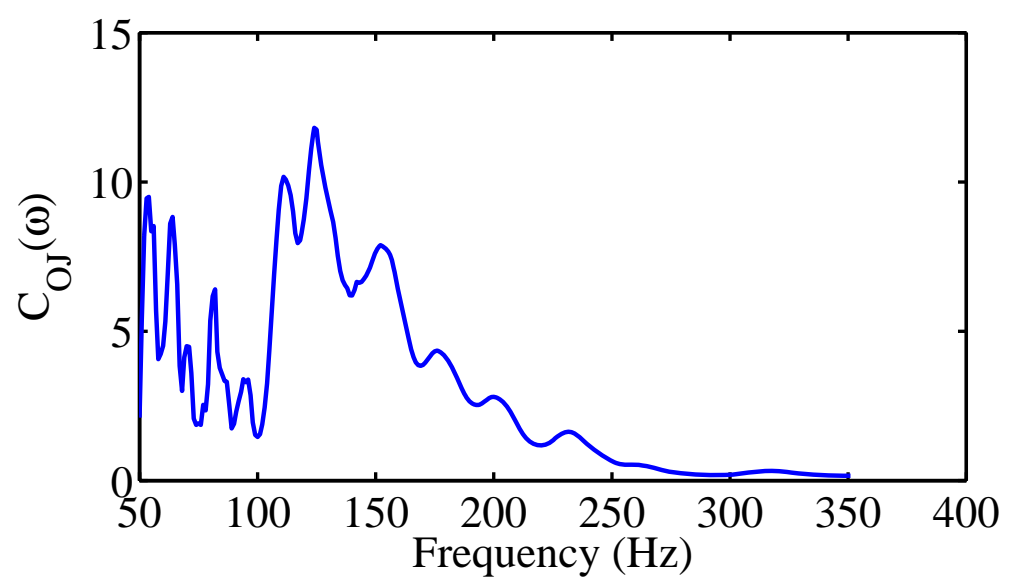

Figure 7. Graph of the partitioning criterion represented by the function $\omega \mapsto C_{O J}(\omega)$ corresponding to the configuration associated with figure 6

(a)

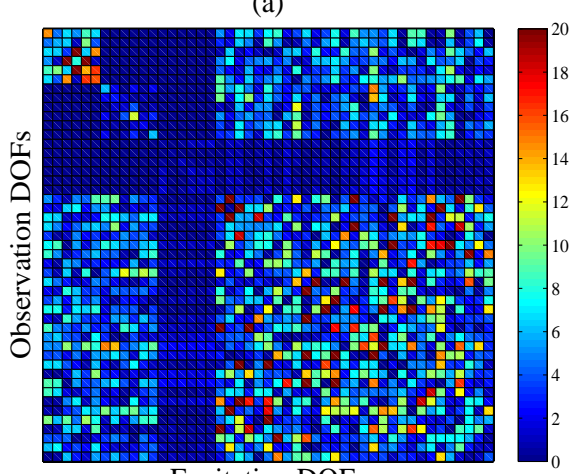

Excitation DOFs

(c)

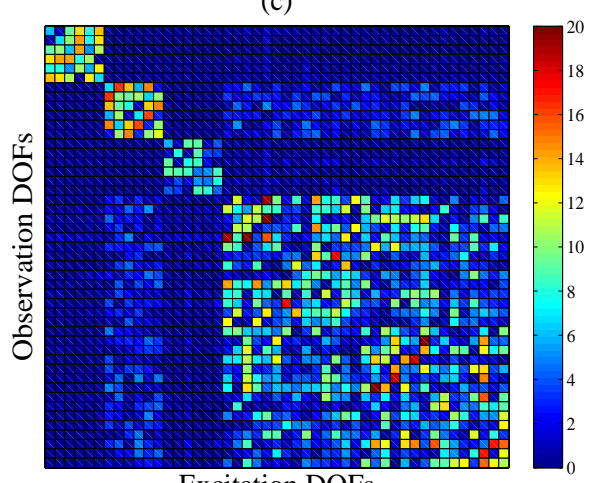

(b)

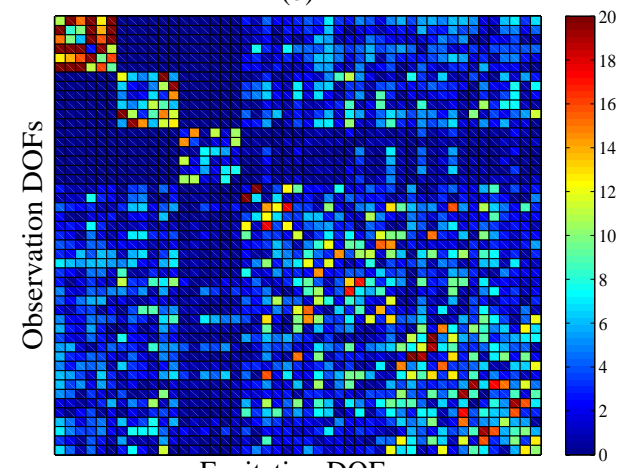

Excitation DOFs

(d)

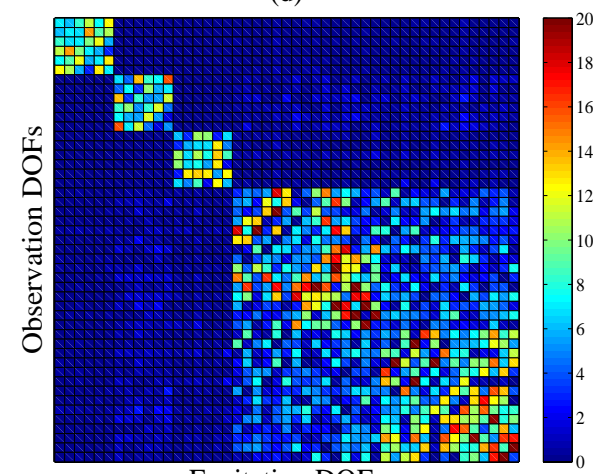

Excitation DOFs

Figure 8. Images of the assembled error matrix $\varepsilon \mathcal{E}^{(\omega)}$ corresponding to all the chosen structural dofs at $50 \mathrm{~Hz}$ (a), $100 \mathrm{~Hz}$ (b), $150 \mathrm{~Hz}$ (c) and $300 \mathrm{~Hz}$ (d). 


\section{Conclusion}

In this paper, a partitioning methodology of complex structures has been presented for the medium-frequency vibroacoustic analysis. This methodology is based on the use of the energy-density field approach introduced in a previous paper. An application to an automotive vehicle has been presented.

First, the construction of the local vibroacoustic energy model and of the simplified local vibroacoustic energy model have been recalled. Then, the extension of the energy-density field approach to the case of general input forces has been explained. A partitioning methodology has then been presented along with a set of tools used for the construction of a partitioning. These tools are (1) an error matrix which gives the error due to the use of the model simplification for a given partitioning and (2) a partitioning criterion which is used to define the zones for which the simplified model can be used.

An application of the partitioning methodology has been carried out for an automotive vehicle. For such a very complex vibroacoustic system, it has been proven that an effective partitioning can be constructed using the components of the vibroacoustic system for all the frequencies larger than a given frequency depending on the degree of the separation. The results show that a partitioning based on components can be observed above $160 \mathrm{~Hz}$. Starting at $50 \mathrm{~Hz}$ the cavity and the rear seat panel are separated while all the other components are coupled together. The roof is then separated at $90 \mathrm{~Hz}$ and finally, the wind shield is separated at approximately $160 \mathrm{~Hz}$. The left and right front suspension and engine supports attachement dofs are separated above $260 \mathrm{~Hz}$.

The method proposed gives an efficient tool in the context of the concurrent engineering for the medium frequency acoustic design process of automotive vehicles. In addition, this methodology could be useful to extend the validity of the SEA to the medium frequency range in defining the SEA subsystems as a partitioning yielding a set of structural subsystems which are not coupled or weakly coupled.

\section{References}

[1] R. Ohayon and C. Soize, Structural Acoustics and Vibrations, Academic Press, San Diego, 1998.

[2] J.F Durand, C. Soize, L. Gagliardini, Structural-acoustic modeling of automotive vehicles in presence of uncertainties and experimental identification and validation, The Journal of the Acoustical Society of America 124(3) (2008) 1513-1525.

[3] C. Fernandez, C. Soize, L. Gagliardini, Fuzzy structure theory modeling 
of sound-insulation layers in complex vibroacoustic uncertain sytems Theory and experimental validation, The Journal of the Acoustical Society of America 125(1) (2009) 138-153.

[4] M. Kassem, C. Soize, L. Gagliardini, Energy density field approach for low- and medium-frequency vibroacoustic analysis of complex structures using a statistical computational model, Journal of Sound and Vibration 323(1) (2009) 849-863.

[5] R.H. Lyon, G. Maidanik, Statistical methods in vibration analysis, AIAA Journal 2(6) (1964) 1015-1024.

[6] R.H. Lyon, Statistical Energy Analysis of Dynamical Systems, MIT Press, San Diego, 1975.

[7] F.J. Fahy, A.D. Mohammed, A study of uncertainty in applications of SEA to coupled beam and plate systems, partI: Computational experiments, Journal of Sound and Vibration 158(1) (1992) 45-67.

[8] L. Maxit, J.-L. Guyader, Extension of SEA model to subsystems with non-uniform modal energy distribution, Journal of Sound and Vibration 265 (2) (2003) 337-358.

[9] N. Totaro, J.L. Guyader, SEA substructuring using cluster analysis: The MIR index, Journal of Sound and Vibration 290(1-2) (2006) 264-289.

[10] P. Ragnarsson, B. Pluymers, S. Donders, W. Desmet, Subcomponent modelling of input parameters for statistical energy analysis by using a wave-based boundary condition, Journal of Sound and Vibration 329(1) (2010) 96-108.

[11] R.S. Langley, P. Bremner, A hybrid method for the vibration analysis of complex structural-acoustic systems, The Journal of the Acoustical Society of America 105(3) (1999) 1657-1671.

[12] P.J. Shorter, R.S. Langley, Vibroacoustic analysis of complex systems, Journal of Sound and Vibration 288(3) (2005) 669-699.

[13] V. Cotoni, R. Langley, P. Shorter, A statistical energy analysis subsystem formulation using finite element and periodic structure theory, Journal of Sound and Vibration 318(4-5) (2008) 1077-1108.

[14] A. LeBot, Energy transfer for high frequencies in built-up structures, Journal of Sound and Vibration 250(2) (2002) 247-275.

[15] R.S. Langley, On the diffuse field reciprocity relationship and vibrational energy variance in a random subsystem at high frequencies, The Journal of the Acoustical Society of America 121(2) (2007) 913-921.

[16] R. Hardy, M. Ichchou, L. Jezequel, D. Trentin, A hybrid local energy formulation for plates mid-frequency flexural vibrations, European Journal of Mechanics A-Solids 28(1) (2009) 121-130.

[17] R.S. Langley, A.W.M. Brown, The ensemble statistics of the bandavaraged energy of random system, Journal of Sound and Vibration 275(3-5) (2004) 847-857.

[18] R.S. Langley, V. Cotoni, Response variance prediction in the statistical energy analysis of built up systems, The Journal of the Acoustical Society of America 115(2) (2004) 706-718. 
[19] V. Cotoni, R.S. Langley, M.R.F. Kinder, Numerical and experimental validation of variance prediction in the statistical energy analysis of builtup systems, Journal of Sound and Vibration 288(3) (2005) 701-728.

[20] G. Orefice, C. Cacciolati, J.L. Guyader, The energy mobility, Journal of Sound and Vibration 254(2) (2002) 269-295.

[21] L. Gagliardini, L. Houillon, G. Borello, L. Petrinelli, Virtual SEA - FEAbased modeling of mid-frequency structure-borne noise, Sound and $V i$ bration 39(1) (2005) 22-28.

[22] C. Soize, Maximum entropy approach for modeling random uncertainties in transient elastodynamics, Journal of Acoustical Society of America 109(5) (2001) 1979-1996.

[23] C. Soize, Random matrix theory for modeling uncertainties in computational mechanics, Computer Methods in Applied Mechanics and Engineering 194(12-16) (2005) 1333-1366.

[24] C. Chen and D. Duhamel and C. Soize, Probabilistic approach for model and data uncertainties and its experimental identification in structural dynamics: Case of composite sandwich panels, Journal of Sound and Vibration 294(1-2) (2006) 64-81.

[25] H. Chebli and C. Soize, Experimental validation of a nonparametric probabilistic model of nonhomogeneous uncertainties for dynamical systems, Journal of Acoustical Society of America 115(2) (2004) 697-705. 